\title{
CLASSIFICATION OF GENERALIZED YAMABE SOLITONS IN EUCLIDEAN SPACES
}

\author{
SHUNYA FUJII AND SHUN MAETA
}

\begin{abstract}
In this paper, we consider generalized Yamabe solitons which include many notions, such as Yamabe solitons, almost Yamabe solitons, $h$-almost Yamabe solitons, gradient $k$-Yamabe solitons and conformal gradient solitons. We completely classify the generalized Yamabe solitons on hypersurfaces in Euclidean spaces arisen from the position vector field.
\end{abstract}

\section{INTRODUCTION}

An $n$-dimensional Riemannian manifold $(M, g)$ is called a Yamabe soliton, if there exist a complete vector field $v$ and $\rho \in \mathbb{R}$ such that

$$
(R-\rho) g=\frac{1}{2} \mathcal{L}_{v} g,
$$

where $R$ is the scalar curvature of $M$ and $\mathcal{L}_{v} g$ is the Lie derivative of $g$. If $v$ is the gradient of some smooth function $f$ on $M$, then $(M, g, f)$ is called a gradient Yamabe soliton. If $f$ is constant, then $(M, g, f)$ is called trivial.

Yamabe solitons are special solutions of the Yamabe flow introduced by R. Hamilton [17]. In the last decade, Yamabe solitons have developed rapidly. To understand the Yamabe soliton, many generalizations of it have been introduced:

(1) Almost Yamabe solitons [2]:

For a complete vector field $v$ and a smooth function $\rho$ on $M$,

$$
(R-\rho) g=\frac{1}{2} \mathcal{L}_{v} g .
$$

(2) Gradient $k$-Yamabe solitons [6]:

2010 Mathematics Subject Classification. 53C25, 53C40, 53C42.

Key words and phrases. Yamabe solitons; almost Yamabe solitons; $k$-Yamabe solitons; $h$-almost Yamabe solitons; Yamabe flow; concurrent vector fields; Hessian manifolds.

The second author is partially supported by the Grant-in-Aid for Young Scientists, No.19K14534, Japan Society for the Promotion of Science. 
For a smooth function $f$ on $M$ and $\rho \in \mathbb{R}$,

$$
2(n-1)\left(\sigma_{k}-\rho\right) g=\nabla \nabla f,
$$

where $\sigma_{k}$ denotes the $\sigma_{k}$-curvature of $g$, that is,

$$
\sigma_{k}=\sigma_{k}\left(g^{-1} A\right)=\sum_{i_{1}<\cdots<i_{k}} \mu_{i_{1}} \cdots \mu_{i_{k}}(\text { for } 1 \leq k \leq n),
$$

where $A=\frac{1}{n-2}\left(\operatorname{Ric}-\frac{1}{2(n-1)} R g\right)$ is the Schouten tensor and $\mu_{1}, \cdots, \mu_{n}$ are the eigenvalues of the symmetric endomorphism $g^{-1} A$. Here, Ric is the Ricci tensor of $M$. We remark that the 1-Yamabe soliton is the Yamabe soliton.

(3) $h$-almost Yamabe solitons [27]:

For a complete vector field $v$ and smooth functions $\rho$ and $h(h>0$ or $h<0)$ on $M$,

$$
(R-\rho) g=\frac{h}{2} \mathcal{L}_{v} g
$$

(4) Conformal gradient solitons [6]:

For smooth functions $f$ and $\varphi$ on $M$,

$$
\varphi g=\nabla \nabla f \text {. }
$$

Many examples of these solitons are known. A warped product manifold $\left(\mathbb{R} \times \cosh t \mathbb{S}^{n}, d t^{2}+\cosh ^{2} t g_{\mathbb{S}^{n}}, f=\sinh t, \rho=\sinh t+n\right)$ is a gradient almost Yamabe soliton, where $\left(\mathbb{S}^{n}, g_{\mathbb{S}^{n}}\right)$ is the standard sphere (cf. [2]). On the cylinder $\mathbb{S}^{n} \times \mathbb{R}$, gradient $k$-Yamabe solitons are constructed (cf. 3]). For a nonzero real number $m$ and a positive constant $\beta,\left(\mathbb{R}^{n}, g_{\text {can }}, f=-m \log \left(|x|^{2}+\beta\right), \rho=\frac{2 m}{|x|^{2}+\beta}\right)$ is a gradient $\left(-\frac{m}{|x|^{2}+\beta}\right)$-almost Yamabe soliton, where $\left(\mathbb{R}^{n}, g_{\text {can }}\right)$ is the Euclidean space (cf. [27]).

Even though there are many examples of these solitons, some classifications are known (cf. [2], [3], [5], 6], [16], [18], [19], [20], 21], [23] and [27]). In particular, there are many results for Yamabe solitons. Any compact Yamabe soliton has constant scalar curvature (cf. [7], [12] and [18]). P. Daskalopoulos and N. Sesum [16] showed that any locally conformally flat complete gradient Yamabe soliton with positive sectional curvature has to be rotationally symmetric. G. Catino, C. Mantegazza and L. Mazzieri [6] classified nontrivial complete gradient Yamabe solitons with nonnegative Ricci tensor. H.-D. Cao, X. Sun and Y. Zhang [5] gave a useful classification. Recently, the second author 21] classified 3-dimensional complete gradient Yamabe solitons with divergence-free Cotton tensor. 
To consider all the generalized Yamabe solitons mentioned above, we consider the following.

Definition 1.1. A Riemannian manifold $(M, g)$ is called a conformal soliton if there exists a complete vector field $v$ such that

$$
\varphi g=\frac{1}{2} \mathcal{L}_{v} g,
$$

for a smooth function $\varphi: M \rightarrow \mathbb{R}$. We denote the conformal soliton by $(M, g, v, \varphi)$. If $v \equiv 0$, then $M$ is called trivial.

Remark 1.2. Conformal solitons include Yamabe solitons, almost Yamabe solitons, gradient $k$-Yamabe solitons, $h$-almost Yamabe solitons and conformal gradient solitons. Therefore, all the results in this paper can be applied to all these solitons.

$v$ is called a conformal vector field.

We can construct many examples of conformal solitons. For example, for a smooth function $f=\log \left(e^{x}+e^{y}+1\right)$ on $\mathbb{R}^{2}$ with coordinate system $\{x, y\}, g=\nabla \nabla f$ is a Riemannian metric on $\mathbb{R}^{2}$. Thus $\left(\mathbb{R}^{2}, g, f, \varphi=1\right)$ is a conformal (gradient) soliton.

To understand conformal solitons, we consider them as hypersurfaces of some Riemannian manifold. As the metric of $M$ is induced from the ambient space, it seems natural to take a soliton vector field $v$ from the ambient space. Let $V$ be some vector field of the ambient space. Then we can decompose $V$ as the tangential component $V^{T}$ and the normal component $V^{\perp}$. Therefore, if the ambient space has a (natural) vector field $V$, it is natural to take $v=V^{T}$. The Euclidean space $\mathbb{E}^{n+1}$ is the most basic and natural one, because it has the position vector field $V$. Some solitons on hypersurfaces in Euclidean spaces or more general manifolds have been studied (cf. [9], [10], [13], [14], [15] and [23]).

In this paper, we completely classify conformal solitons on a hypersurface in the Euclidean space $\mathbb{E}^{n+1}$ arisen from the position vector field.

Theorem 1.3. Any conformal soliton $\left(M, g, V^{T}, \varphi\right)$ on a hypersurface in the Euclidean space $\mathbb{E}^{n+1}$ is contained in a hyperplane, a conic hypersurface or a hypersphere.

A submanifold $M^{n}$ in the Euclidean space $\mathbb{E}^{m}$ is called a conic submanifold if it is an open portion of a cone with vertex at the origin (cf. [8]). Here we remark that by the definition, $n$-dimensional planes through the origin are included in $n$-dimensional conic submanifolds.

The following fact is used later. 
Proposition $1.4([8])$. Let $f: M \rightarrow \mathbb{E}^{m}$ be an isometric immersion of an n-dimensional Riemannian manifold into the m-dimensional Euclidean space $\mathbb{E}^{m}$. Then $V=V^{T}$ holds identically if and only if $M$ is a conic submanifold.

\section{Preliminaries}

Let $(N, \tilde{g})$ be an $m$-dimensional Riemannian manifold and $(M, g)$ be an $n$-dimensional submanifold in $(N, \tilde{g})$. All manifolds in this paper are assumed to be smooth, orientable and connected. We denote LeviCivita connections on $(M, g)$ and $(N, \tilde{g})$ by $\nabla$ and $\tilde{\nabla}$, respectively. The Lie derivative of $g$ is defined by

$$
\mathcal{L}_{X} g(Y, Z)=X(g(Y, Z))-g([X, Y], Z)-g(Y,[X, Z]),
$$

for any vector fields $X, Y, Z$ on $M$.

For any vector fields $X, Y$ tangent to $M$ and $\eta$ normal to $M$, the formula of Gauss is given by

$$
\tilde{\nabla}_{X} Y=\nabla_{X} Y+h(X, Y),
$$

where $\nabla_{X} Y$ and $h(X, Y)$ are the tangential and the normal components of $\tilde{\nabla}_{X} Y$. The formula of Weingarten is given by

$$
\tilde{\nabla}_{X} \eta=-A_{\eta}(X)+D_{X} \eta
$$

where $-A_{\eta}(X)$ and $D_{X} \eta$ are the tangential and the normal components of $\tilde{\nabla}_{X} \eta . A_{\eta}(X)$ and $h(X, Y)$ are related by

$$
g\left(A_{\eta}(X), Y\right)=\tilde{g}(h(X, Y), \eta) .
$$

The mean curvature vector $H$ of $M$ in $N$ is given by

$$
H=\frac{1}{n} \text { trace } h \text {. }
$$

For any vector fields $X, Y, Z, W$ tangent to $M$, the equation of Gauss is given by

$$
\begin{aligned}
\tilde{g}(\tilde{\operatorname{Rm}}(X, Y) Z, W)= & g(R m(X, Y) Z, W) \\
& +\tilde{g}(h(X, Z), h(Y, W)) \\
& -\tilde{g}(h(X, W), h(Y, Z)),
\end{aligned}
$$

where $R m$ and $\tilde{R m}$ are Riemannian curvature tensors of $M$ and $N$, respectively. The equation of Codazzi is given by

$$
(\tilde{R m}(X, Y) Z)^{\perp}=\left(\bar{\nabla}_{X} h\right)(Y, Z)-\left(\bar{\nabla}_{Y} h\right)(X, Z)
$$


where $(\tilde{R m}(X, Y) Z)^{\perp}$ is the normal component of $\tilde{R m}(X, Y) Z$ and $\bar{\nabla}_{X} h$ is defined by

$$
\left(\bar{\nabla}_{X} h\right)(Y, Z)=D_{X} h(Y, Z)-h\left(\nabla_{X} Y, Z\right)-h\left(Y, \nabla_{X} Z\right) .
$$

If $N$ is a space of constant curvature, then the equation of Codazzi reduces to

$$
0=\left(\bar{\nabla}_{X} h\right)(Y, Z)-\left(\bar{\nabla}_{Y} h\right)(X, Z)
$$

\section{Conformal solitons With a CONCURREnt VeCtor Field}

The position vector field $V$ on Euclidean spaces satisfies

$$
\nabla_{X} V=X
$$

for any vector field $X$. We consider one of the generalizations of the position vector field, namely, a concurrent vector field.

Definition 3.1. A vector field $V$ on $M$ is called a concurrent vector field if it satisfies

$$
\nabla_{X} V=X
$$

for any vector field $X$ on $M$.

There are several studies of concurrent vector fields (see for example [4], 25] and [26]).

In this section, we consider a conformal soliton with a concurrent vector field.

Firstly, we show a useful formula for study of conformal solitons.

Lemma 3.2. Let $(M, g, f, \varphi)$ be a conformal gradient soliton. Then, we have

$$
(n-1) \Delta \varphi+\frac{1}{2} g(\nabla R, \nabla f)+R \varphi=0
$$

Proof. Since

$$
\begin{gathered}
\Delta \nabla_{i} f=\nabla_{i} \Delta f+R_{i j} \nabla_{j} f, \\
\Delta \nabla_{i} f=\nabla_{k} \nabla_{k} \nabla_{i} f=\nabla_{k}\left(\varphi g_{k i}\right)=\nabla_{i} \varphi
\end{gathered}
$$

and

$$
\nabla_{i} \Delta f=\nabla_{i}(n \varphi)=n \nabla_{i} \varphi
$$

we have

$$
(n-1) \nabla_{i} \varphi+R_{i j} \nabla_{j} f=0,
$$

where $R_{i j}$ is the Ricci tensor of $M$. By applying $\nabla_{l}$ to the both sides of (4), we obtain

$$
(n-1) \nabla_{l} \nabla_{i} \varphi+\nabla_{l} R_{i j} \cdot \nabla_{j} f+R_{i j} \nabla_{l} \nabla_{j} f=0 .
$$

Taking the trace, we obtain (3). 
Proposition 3.3. Any conformal soliton $(M, g, v, \varphi)$ which has a concurrent vector field $v$ is a conformal gradient soliton with $\varphi=1$.

Proof. Since $v$ is a concurrent vector field, we have

$$
g(v, X)=g\left(v, \nabla_{X} v\right)=X\left(\frac{1}{2} g(v, v)\right)
$$

and

$$
\begin{aligned}
\mathcal{L}_{v} g(X, Y) & =v g(X, Y)-g([v, X], Y)-g(X,[v, Y]) \\
& =v g(X, Y)-v g(X, Y)+g\left(\nabla_{X} v, Y\right)+g\left(X, \nabla_{Y} v\right) \\
& =2 g(X, Y),
\end{aligned}
$$

for any vector fields $X, Y$ on $M$. By putting $f=\frac{1}{2} g(v, v)$ on the equation (6), we obtain $v=\nabla f$. Substituting (17) into (2), we have

$$
\varphi=1
$$

From Proposition 3.3, the equation of the conformal soliton with a concurrent vector field is as follows:

$$
g=\nabla \nabla f
$$

Therefore $g$ should be a Hessian metric. The Hessian metric is an important notion on Geometry and Physics (cf. [1], [11], 22] and [24]).

Example 1. A Hessian manifold $(M, g)$ is a conformal gradient soliton with $\varphi=1$.

If $M$ is compact, then there exist no non trivial conformal solitons with a concurrent vector field.

Corollary 3.4. There exists no compact conformal soliton such that the conformal vector field is a concurrent vector field.

Proof. By Proposition 3.3 and (2), we have $\Delta f=n$. By applying maximum principle, we get $f$ is constant, which cannot happen.

\section{Conformal solitons on SUBmanifolds}

In this section, we assume that $(N, \tilde{g})$ is a Riemannian manifold endowed with a concurrent vector field $V$ and $(M, g)$ is a submanifold in $(N, \tilde{g}) . V^{T}$ and $V^{\perp}$ denote the tangential and the normal components of $V$, respectively.

Firstly, we show the following lemma which will be used later for the purpose of classification of the conformal solitons. 
Lemma 4.1. Any conformal soliton $\left(M, g, V^{T}, \varphi\right)$ on a submanifold $M$ in $N$ satisfies

$$
(\varphi-1) g(X, Y)=g\left(A_{V^{\perp}}(X), Y\right)
$$

for any vector fields $X, Y$ on $M$.

Proof. From the definition of the Lie derivative, we have

$$
\begin{aligned}
\left(\mathcal{L}_{V^{T}} g\right)(X, Y) & =V^{T} g(X, Y)-g\left(\nabla_{V^{T}} X-\nabla_{X} V^{T}, Y\right)-g\left(X, \nabla_{V^{T}} Y-\nabla_{Y} V^{T}\right) \\
& =g\left(\nabla_{X} V^{T}, Y\right)+g\left(X, \nabla_{Y} V^{T}\right) \\
& =\tilde{g}\left(\tilde{\nabla}_{X} V-\tilde{\nabla}_{X} V^{\perp}, Y\right)+\tilde{g}\left(X, \tilde{\nabla}_{Y} V-\tilde{\nabla}_{Y} V^{\perp}\right) \\
& =2 g(X, Y)+2 g\left(A_{V^{\perp}}(X), Y\right),
\end{aligned}
$$

for any vector fields $X, Y$ on $M$. Combining (9) with (2), we obtain (8).

Proposition 4.2. Any conformal soliton $\left(M, g, V^{T}, \varphi\right)$ on a submanifold $M$ in $N$ is a conformal gradient soliton.

Proof. Set

$$
f=\frac{1}{2} \tilde{g}(V, V)
$$

For any vector field $X$ on $M$, we obtain

$$
g\left(V^{T}, X\right)=\tilde{g}(V, X)=\tilde{g}\left(V, \tilde{\nabla}_{X} V\right)=X\left(\frac{1}{2} \tilde{g}(V, V)\right)=X f=g(\nabla f, X) .
$$

Proposition 4.3. If a conformal soliton $\left(M, g, V^{T}, \varphi\right)$ on a submanifold $M$ in $N$ is minimal, then $\varphi=1$.

Proof. Let $\left\{e_{1}, \cdots, e_{n}\right\}$ be an orthonormal frame on $M$. By Lemma4.1, we have

$$
(\varphi-1) g_{i j}=g\left(A_{V^{\perp}}\left(e_{i}\right), e_{j}\right)=\tilde{g}\left(h\left(e_{i}, e_{j}\right), V^{\perp}\right) .
$$

Since $M$ is minimal and taking the trace, we obtain

$$
n(\varphi-1)=n \tilde{g}\left(H, V^{\perp}\right)=0 .
$$

Therefore, we conclude that

$$
\varphi=1
$$

Corollary 4.4. There exists no compact conformal soliton on a minimal submanifold in $N$ such that the conformal vector field is $V^{T}$.

Proof. By Proposition 4.3 and (2), we have $\Delta f=n$. By applying maximum principle, we get $f$ is constant, which cannot happen. 


\section{Proof of Theorem 1.3}

We hereafter denote $V$ by the position vector field of $\mathbb{E}^{n+1}$. In this section, we give the proof of Theorem 1.3 as follows.

Theorem 5.1. Any conformal soliton $\left(M, g, V^{T}, \varphi\right)$ on a hypersurface in the Euclidean space $\mathbb{E}^{n+1}$ is contained in a hyperplane, a conic hypersurface or a hypersphere.

Proof. Let $\alpha$ be a mean curvature and $\lambda$ be a support function of $M$, i.e., $H=\alpha N$ and $\lambda=\tilde{g}(N, V)$ with a unit normal vector field $N$. Set $U_{0}=\{x \in M \mid \lambda=0\}$. From Lemma 4.1, we have

$$
(\varphi-1) g_{i j}=\tilde{g}\left(h\left(e_{i}, e_{j}\right), V^{\perp}\right)=\tilde{g}\left(\kappa_{i} g_{i j} N, V\right)=\kappa_{i} g_{i j} \lambda,
$$

where $A_{N}\left(e_{i}\right)=\kappa_{i} e_{i},(i=1, \cdots, n)$. Hence we have

$$
\varphi-1=\lambda \kappa_{i} \text {. }
$$

Let $f$ be a smooth function on $M$ defined by

$$
f(x):=\prod_{i=1}^{n} \kappa_{i}(x) \quad x \in M .
$$

Case 1. $U_{0}=\emptyset$ : By taking the summation, we obtain

$$
\varphi-1=\lambda \alpha \text {. }
$$

Comparing (10) and (11), we have

$$
\kappa_{i}=\alpha .
$$

Thus $M$ is totally umbilical with $A_{N}\left(e_{i}\right)=\alpha e_{i}$ and $h$ satisfies $h(X, Y)=$ $\alpha g(X, Y) N$. Since $N$ is a unit normal vector field, we have

$$
0=\tilde{\nabla}_{X}(\tilde{g}(N, N))=2 \tilde{g}\left(\tilde{\nabla}_{X} N, N\right)=2 \tilde{g}\left(D_{X} N, N\right) .
$$

Therefore, $D_{X} N=0$. Hence we obtain

$$
\begin{aligned}
\left(\bar{\nabla}_{X} h\right)(Y, Z) & =D_{X} h(Y, Z)-h\left(\nabla_{X} Y, Z\right)-h\left(Y, \nabla_{X} Z\right) \\
& =X(\alpha) g(Y, Z) N,
\end{aligned}
$$

for any vector fields $X, Y, Z$ on $M$. From the equation of Codazzi, we have

$$
X(\alpha) Y=Y(\alpha) X
$$

Since we can assume that $X$ and $Y$ are linearly independent, we conclude that $\alpha$ and $f$ are constant respectively.

If $\alpha=0$, then by $\tilde{\nabla}_{X} N=0, N$, restricted to $M$, is a constant vector field in $\mathbb{E}^{n+1}$ and we have

$$
\tilde{\nabla}_{X}(\tilde{g}(V, N))=\tilde{g}\left(\tilde{\nabla}_{X} V, N\right)+\tilde{g}\left(V, \tilde{\nabla}_{X} N\right)=\tilde{g}(X, N)=0 .
$$


This shows that $\lambda=\tilde{g}(V, N)$ is constant when $V$ and $N$ are restricted to $M$. Therefore, $M$ is contained in a hyperplane normal to $N$ which does not through the origin and $\varphi=1$.

If $\alpha \neq 0$, then we have

$$
\tilde{\nabla}_{X}\left(V+\alpha^{-1} N\right)=X+\alpha^{-1} \tilde{\nabla}_{X} N=X+\alpha^{-1}\left(-A_{N}(X)\right)=0 .
$$

This shows that the vector field $V+\alpha^{-1} N$, restricted to $M$, is a constant one in $\mathbb{E}^{n+1}$. Therefore, $M$ is contained in a hypersphere.

Case 2. $U_{0}=M$ :

We have $V=V^{T}$. By Proposition 1.4, we obtain that $M$ is contained in a conic hypersurface.

Case 3. Others:

Take $p \in M \backslash U_{0}$, that is, $\lambda \neq 0$ on some open set $\Omega \ni p$. By the same argument as in Case 1, we have $\Omega$ is an open portion of a hyperplane or a hypersphere.

We consider the case that $\Omega$ is an open portion of a hyperplane. Without loss of generality, we can take $\Omega$ as the maximum connected component which is an open set including $p$ on $M \backslash U_{0}$. On $\Omega, \lambda=$ $\tilde{g}(V, N)(\neq 0)$ is constant, say $\lambda_{\Omega}$. Since $M$ is connected, if $\Omega$ is closed, then $\Omega=M$, which is a contradiction. If $M$ is not closed, then we have $\partial \Omega \neq \emptyset$ and $\partial \Omega \cap \Omega=\emptyset$. Take $q \in \partial \Omega$. Since $\lambda$ is continuous, we have $\lambda(q)=\lambda_{\Omega}$. Thus we can take an open neighborhood $U_{q}$ of $q$ such that $\lambda \neq 0$ on $U_{q}$. Since $\Omega$ is the maximum connected component, we have a contradiction. Hence, we have that $\Omega$ is an open portion of a hypersphere.

If Int $U_{0} \neq \emptyset$, for any $x \in \operatorname{Int} U_{0}$, we can take some open set $U_{x} \ni x$ which is included in $U_{0}$. Let $\left\{e_{i}\right\}$ be an orthonormal frame defined on $U_{x}$ which satisfies

$$
A_{N}\left(e_{i}\right)(x)=\kappa_{i}(x) e_{i}(x)
$$

and

$$
\nabla_{e_{i}} e_{j}(x)=0, \quad 1 \leq i, j \leq n .
$$

On $U_{0}$, we have $V=V^{T}$. Since $V$ is a concurrent vector field,

$$
X=\tilde{\nabla}_{X} V^{T}=\nabla_{X} V^{T}+h\left(X, V^{T}\right),
$$

on $\operatorname{Int} U_{0}$.

So we obtain

$$
\nabla_{X} V^{T}=X
$$


and

$$
h\left(X, V^{T}\right)=0
$$

From (14),

$$
\begin{aligned}
e_{i}(x) & =\nabla_{e_{i}} V^{T}(x) \\
& =\nabla_{e_{i}}\left(V^{T}\right)^{j} e_{j}(x) \\
& =e_{i}\left(V^{T}\right)^{j}(x) e_{j}(x)+\left(V^{T}\right)^{j}(x) \nabla_{e_{i}} e_{j}(x) .
\end{aligned}
$$

Since $\nabla_{e_{i}} e_{j}(x)=0$,

$$
e_{i}(x)=e_{i}\left(V^{T}\right)^{j}(x) e_{j}(x) .
$$

Therefore,

$$
e_{i}\left(V^{T}\right)^{j}(x)=\delta_{i j}
$$

From (15),

$$
\begin{aligned}
0 & =\tilde{g}\left(h\left(e_{i}, V^{T}\right), N\right) \\
& =g\left(A_{N}\left(e_{i}\right), \sum_{j=1}^{n}\left(V^{T}\right)^{j} e_{j}\right) \\
& =\sum_{j=1}^{n}\left(V^{T}\right)^{j} \tau_{i j},
\end{aligned}
$$

where $\tau_{i j}$ is a smooth function defined by $\tau_{i j}:=g\left(A_{N}\left(e_{i}\right), e_{j}\right)$ on $U_{x}$. From (16) and (17),

$$
\begin{aligned}
0 & =e_{i}\left(\sum_{j=1}^{n}\left(V^{T}\right)^{j} \tau_{i j}\right)(x) \\
& =\sum_{j=1}^{n}\left\{e_{i}\left(\left(V^{T}\right)^{j}\right)(x) \tau_{i j}(x)+\left(V^{T}\right)^{j}(x) e_{i}\left(\tau_{i j}\right)(x)\right\} \\
& =\kappa_{i}(x)+\sum_{j=1}^{n}\left(V^{T}\right)^{j}(x) e_{i}\left(\tau_{i j}\right)(x) .
\end{aligned}
$$

If $f(x) \neq 0$, we obtain $\left(V^{T}\right)^{j}(x)=0 \quad(1 \leq j \leq n)$ from (17). This contradicts (18) and $f(x) \neq 0$. So $f=0$ and $X(f)=0$ on $\operatorname{Int} U_{0}$ for any vector field $X$ on $M$. From Case $1, X(f)=0$ on $M \backslash U_{0}$. This 
means $X(f)=0$ on $M$. So we conclude that $f$ is constant on $M$. But $f \neq 0$ on $M \backslash U_{0}$, this is a contradiction. Therefore, $\operatorname{Int} U_{0}=\emptyset$.

By the same argument as in Case $1, \kappa_{i}=\kappa_{j}$ and $X\left(\kappa_{i}\right)=0$ on $M \backslash U_{0}$ for any $1 \leq i, j \leq n$ and any vector field $X$. From this and $\operatorname{Int} U_{0}=\emptyset$, $\kappa_{i}$ is constant which doesn't depend on $i$ on $M$.

Therefore, $M$ is contained in a hypersphere.

\section{REFERENCES}

1. S. Amari and J. Armstrong, Curvature of Hessian manifolds, Diff. Geom. Appl. 33 (2014), 1-12.

2. E. Barbosa and E. Ribeiro, On conformal solutions of the Yamabe flow, Arch. Math. 101 (2013), 79-89.

3. L. Bo, P. T. Ho and W. Sheng, The $k$-Yamabe solitons and the quotient Yamabe solitons, Nonlinear Anal. 168 (2018), 181-195.

4. F. Brickell and K. Yano, Concurrent vector fields and Minkowski structures, Kodai Math. Sem. rep. 26 (1974), 22-28.

5. H.-D. Cao, X. Sun and Y. Zhang, On the structure of gradient Yamabe solitons, Math. Res. Lett. 19 (2012), 767-774.

6. G. Catino, C. Mantegazza and L. Mazzieri, On the global structure of conformal gradient solitons with nonnegative Ricci tensor, Comm. Contempt. Math. 14 (2012), 12pp.

7. L. F. D. Cerbo and M. M. Disconzi, Yamabe Solitons, Determinant of the Laplacian and the Uniformization Theorem for Riemann Surfaces, Lett. Math. Phys. 83 (2008), 13-18.

8. B. Y. Chen Differential geometry of rectifying submanifolds, Int. Electron. J. Geom. 9 (2016), 1-8.

9. B. Y. Chen and S. Deshmukh, Ricci solitons and concurrent vector fields, Balkan J. Geom. Appl. 20 (2015), no. 1, 14-25.

10. B. Y. Chen and S. Deshmukh, Yamabe and quasi-Yamabe solitons on Euclidean submanifolds, Mediterr. J. Math. 15 (2018), 9 pp.

11. S. Y. Cheng, S. T. Yau, The real Monge-Ampére equation and affine flat structures, in: Proceedings of the 1980 Beijing Symposium on Differential Geometry and Differential Equations, Vol.1-3, Beijing, (1980), 339-370.

12. B. Chow, P. Lu and L. Ni, Hamilton's Ricci Flow, Graduate Studies in Mathematics 77, Amer. Math. Soc., (2006).

13. J. T. Cho and M. Kimura, Ricci solitons and real hypersurfaces in a complex space form, Tohôku Math. J. 61 (2009), 205-212.

14. J. T. Cho and M. Kimura, Ricci solitons of compact real hypersurfaces in Kähler manifolds, Math. Nachr. 284 (2011), 1385-1393.

15. J. T. Cho and M. Kimura, Ricci solitons on locally conformally flat hypersurfaces in space forms, J. Geom. Phys. 62 (2012), 1882-1891.

16. P. Daskalopoulos, and N. Sesum, The classification of locally conformally flat Yamabe solitons, Adv. Math. 240 (2013), 346-369.

17. R. Hamilton, Lectures on geometric flows, (1989), unpublished.

18. S. Y. Hsu, A note on compact gradient Yamabe solitons, J. Math. Anal. Appl. 388 (2) (2012) 725-726. 
19. L. Ma and V. Miquel, Remarks on scalar curvature of Yamabe solitons, Ann. Glob. Anal. Geom. 42 (2012), 195-205 DOI 10.1007/s10455-011-9308-7.

20. S. Maeta, Complete Yamabe solitons with finite total scalar curvature, Diff. Geom. Appl. 66 (2019), 75-81.

21. S. Maeta, Three-dimensional complete gradient Yamabe solitons with divergence-free Cotton tensor, Ann. Glob. Anal. Geom. 58 (2020), 227-237.

22. M. Mirghafouri and F. Malek, Long-time existence of a geometric flow on closed Hessian manifolds, J. Geom. Phys. 110 (2017), 54-65.

23. T. Seko and S. Maeta, Classification of almost Yamabe solitons in Euclidean spaces, J. Geom. Phys. 136 (2019), 97-103.

24. H. Shima and K. Yagi, Geometry of Hessian manifolds, Diff. Geom. Appl. 7 (1997), 277-290.

25. K. Yano, Sur le parallélisme et la concourance dans l'espace de Riemann, Proc. Imp. Acad. Tokyo, 19 (1943), 189-197.

26. K. Yano and B. Y. Chen, On the concurrent vector fields of immersed manifolds, Kodai Math. Sem. Rep. 23 (1971), 343-350.

27. F. Zeng, On the h-almost Yamabe soliton J. Math. Study (to appear).

Department of Mathematics, Shimane University, Nishikawatsu 1060 Matsue, 690-8504, JaPAN.

Email address: shunyaf3@gmail.com

Department of Mathematics, Shimane University, Nishikawatsu 1060 MATSUE, 690-8504, JAPAN.

Email address: shun.maeta@gmail.com or maeta@riko.shimane-u.ac.jp 